Preprint CAMTP/99-1

March 1999

\title{
Study of regular and irregular states in generic systems
}

\author{
Gregor Veble, Marko Robnik and Junxian Liu 1 \\ Center for Applied Mathematics and Theoretical Physics, \\ University of Maribor, Krekova 2, SI-2000 Maribor, Slovenia
}

\begin{abstract}
In this work we present the results of a numerical and semiclassical analysis of high lying states in a Hamiltonian system, whose classical mechanics is of a generic, mixed type, where the energy surface is split into regions of regular and chaotic motion. As predicted by the principle of uniform semiclassical condensation (PUSC), when the effective $\hbar$ tends to 0 , each state can be classified as regular or irregular. We were able to semiclassically reproduce individual regular states by the EBK torus quantization, for which we devise a new approach, while for the irregular ones we found the semiclassical prediction of their autocorrelation function, in a good agreement with numerics. We also looked at the low lying states to better understand the onset of semiclassical behaviour.
\end{abstract}

PACS numbers: 02.50.+s, 03.65.-w, 03.65.Ge, 03.65.Sq, 05.40.+j, 05.45.+b

Submitted to Journal of Physics A: Mathematical and General

\footnotetext{
${ }^{1}$ e-mails: robnik@uni-mb.si, gregor.veble@uni-mb.si. junxian.liu@uni-mb.si
} 


\section{Introduction}

There is a remarkable difference between the quantum properties of the classically integrable and fully chaotic (ergodic) systems. While in the integrable case the wavefunctions possess an ordered structure, the eigenstates of classically ergodic systems appear random (Berry 1977, Voros 1979) in the semiclassical limit $\hbar \rightarrow 0$. They can be locally well represented as a superposition of plane waves with equal wavevector magnitude but random phases, leading to the Gaussian distribution of the wavefunction amplitude. The assumption of random phases may, however, break down on dynamical grounds when $\hbar$ is not sufficiently small. This leads to the phenomenon of scars, which are the regions of amplified wavefunction amplitude close to the short and weakly unstable classical periodic orbits (Heller 1984).

In the classically mixed systems the energy (hyper)surface is split into both chaotic regions, within which the motion is ergodic, and the regular regions where motion is, as in the fully integrable case, quasiperiodic and confined to invariant tori within the energy surface. We call such systems generic since this is the most general type of Hamiltonian dynamics. The principle of uniform semiclassical condensation (PUSC, see Robnik 1988,1998) states that the quantal phase space distribution of any eigenstate, given by its Wigner function, should be uniformly distributed on a classically invariant object in the phase space when $\hbar \rightarrow 0$. This object can be either an invariant torus in one of the regular regions in the phase space or a whole chaotic component.

While the PUSC is valid for fully integrable and ergodic systems, its full potential is shown when applied to the generic case. Here it predicts that the eigenstates are separated into regular and irregular ones, depending on whether the classical object onto which their Wigner function condenses is a regular torus or a chaotic component, respectively. This has far reaching consequences leading, for example, to the picture of Berry and Robnik (1984) for the statistics of energy levels, where the regular and chaotic states are assumed to contribute independent level sequences to the total spectrum. This was confirmed by many numerical computations (Prosen and Robnik 1993b, 1994 and 1999, Prosen 1995, 1996 and 1998, Robnik 1998).

In this work we are interested in geometrical and statistical properties of high lying eigenfunctions. It is an extension of previous work done on mixed type systems by Prosen and Robnik $(1993 \mathrm{c}, 1994)$ and by Li and Robnik (1995a,b), but also relates to and further develops the paper by Li and 
Robnik (1994) concerning the statistical properties of chaotic states, which drastically differ from the properties of regular states in classically integrable systems (Robnik and Veble 1998). The main step forward in the numerical direction was the use of the so-called scaling method, introduced by Vergini and Saraceno (1995), with which we obtained the states with consecutive indices around $2.5 \cdot 10^{6}$ and thus it enabled us to go much farther into the semiclassical region. On the other hand, we were able to semiclassically reconstruct the numerically obtained regular states by employing the EinsteinBrillouin-Keller (EBK) torus quantization in section 3.1, where we offer a new approach to this problem, especially on the numerical side. While we were not able to semiclassically reconstruct the chaotic states, we obtained a good prediction of their statistical properties by strictly employing the PUSC (see Robnik 1998) in section 3.2.

\section{Our catalogues of states}

We dealt with a model billiard system obtained by conformally mapping the unit circle with the complex quadratic polynomial, as introduced by Robnik (1983, 1984),

$$
z \rightarrow w(z)=z+\lambda z^{2}, w(z)=x+i y .
$$

The range of $x$ at $y=0$ inside the billiard is $x \in[-1+\lambda,+1+\lambda]$. We used the value of $\lambda=0.15$, where the classical phase space is roughly equally divided into components of regular and chaotic motion. We chose the Poincaré surface of section (SOS) to lie on the symmetry axis $y=0$ with coordinate $x$ and the conjugate momentum $p_{x}$ as the parameters of the surface. The intersection of the main chaotic component of our billiard with the SOS is shown in the figure 1 . The coordinate $x$ is taken relative to the center of the billiard (i.e. it is shifted by $\lambda$ w.r.t. $x=0$ of equation (1), so that now the range of $x$ is $x \in[-1,+1]$ ), while $p_{x}$ is the $x$-component of the unit momentum vector.

The quantum mechanics of billiards is described by the Helmholtz equation

$$
\left(\Delta+k^{2}\right) \psi=0
$$

with the Dirichlet boundary conditions, where $k^{2}=2 m E / \hbar^{2}$. We limited 
ourselves to the states with even parity with respect to reflection across the symmetry line $y=0$.

For each state we calculated the smoothed projection of the Wigner function. The Wigner function of a state $\psi(\mathbf{q})$ in the general case of $N$ degrees of freedom is defined in the full phase space (q, p) as (Berry 1983)

$$
W(\mathbf{q}, \mathbf{p})=\frac{1}{(2 \pi \hbar)^{N}} \int d^{N} \mathbf{X} \exp (-i \mathbf{p} \cdot \mathbf{X} / \hbar) \psi^{\dagger}(\mathbf{q}-\mathbf{X} / 2) \psi(\mathbf{q}+\mathbf{X} / 2) .
$$

In our case the eigenfunctions $\psi(x, y)$ generate their Wigner transforms $W\left(x, y, p_{x}, p_{y}\right)$ through (3), where $N=2$. In order to compare the Wigner function of a state of our system with the classical SOS plot we took its value on the symmetry line $(y=0)$ and integrated it over $p_{y}$,

$$
\rho_{S O S}\left(x, p_{x}\right)=\int d p_{y} W\left(x, y=0, p_{x}, p_{y}\right) .
$$

The result is

$\rho_{S O S}\left(x, p_{x}\right)=\frac{1}{2 \pi \hbar} \int d X \exp \left(-i p_{x} X / \hbar\right) \psi^{\dagger}(x-X / 2, y=0) \psi(x+X / 2, y=0)$.

Here we see the reason for considering the even parity states only, because $\psi(x, y=0)$ is exactly zero for odd states, and therefore a different approach must be used to analyze them.

As is well known, the Wigner function is not positive definite but exhibits small oscillations that can blur the overall picture. We chose to smooth the projection of the Wigner function by a suitable Gaussian. It was chosen narrower than the minimum uncertainty Gaussian (in which case the Wigner function becomes the positive definite Husimi distribution) in order not to smooth out too many features, but still wide enough to reduce the oscillations.

The first catalogue of eigenstates and the corresponding smoothed Wigner function projections comprises the first 1000 even states. They were obtained by the conformal mapping diagonalization technique (as described in Robnik 1984). We stress that by this method no levels and eigenstates were lost, as can happen with other approaches such as Heller's plane wave decomposition method (Heller 1984) and/or the boundary integral method (see e.g. Berry and Wilkinson 1984) etc. 
Such a complete catalogue gives us a good picture of the overall behaviour of the system. Many of the states in this low energy region can be associated with the shortest classical periodic orbits. In a mixed type system such as ours, there are both stable orbits that are found within the islands of stability, or unstable ones that lie within one of the chaotic components.

The shortest periodic orbits of our system are shown in figure 2 . These are the stable (labeled by 1) and unstable (5) periodic orbit with two bounces, the stable (2) and unstable (6) three bounce periodic orbit and the stable periodic orbit with four bounces (4). As our billiard is convex, there also exists an infinity of stable periodic orbits skipping along the boundary (3) of the billiard, which are associated with and support the whispering gallery modes (Lazutkin 1981,1991, Li and Robnik 1995a).

Examples of the states that correspond to the stable periodic orbits with the indices (1-4) in the figure 2 are shown in the corresponding rows of the figure 3 with the smoothed projections of their Wigner functions shown in the figure 5. Each row shows the states of the same type with increasing energy. The states corresponding to the stable periodic orbits appear consistently and systematically across the catalogue of states. As we will see later, they can be attributed to the quantized tori in the regular regions, and can be found for those tori whose actions satisfy the Einstein-Brillouin-Keller quantization condition (see Robnik 1998). If we compare the Wigner plots of these states with the SOS plot in figure 1, we notice that the areas of greatest intensities of Wigner functions are found within the corresponding islands of stability in the SOS plot.

The states that correspond to the unstable periodic orbits with indices (5) and (6) are shown in the rows of the figure 1 with the corresponding smoothed projections of their Wigner functions given in the figure 6, with their energy again increasing along the rows. The states corresponding to the unstable periodic orbits emerge with varying intensities with respect to the background, and are less frequent with increasing energy. The approximate position of their emergence in the spectrum can be determined by the condition that the classical action along the periodic orbit should be a multiple of Planck's constant plus the Maslov's phase corrections due to the caustics formed by nearby trajectories (Robnik 1989). Therefore, in 2-dim billiards, the intervals (either in energy $E$ or the cumulative number of states $\mathcal{N}$ ) of consecutive re-appearance of the eigenstates of the same type grow as the square root of $E$ or $\mathcal{N}$. Such states with increased intensities of amplitude 
close to the classically unstable periodic orbits are called scars (Heller 1984, 1986, Bogomolny 1988).

With increasing energy the longer periodic orbits start to manifest themselves in the structure of eigenstates, however, many of the states are becoming increasingly difficult to associate with simple periodic orbits. Two examples of such states are shown in figure 01. The left state is a regular state spanned by a torus in the neighbourhood of the stable periodic orbit with three bounces, characterised by two quantum numbers. It is interesting to note that while the quantum number along the direction of the periodic orbit is quite large (about 140), the transversal quantum number is equal to 1 , giving rise to a single nodal line along the direction of the periodic orbit. On the other hand the state on the right can not be associated only with a single unstable periodic orbit, but with a large portion of the chaotic component, this being more prominent in the smoothed projections of the corresponding Wigner function shown in the lower row of the figure 7 .

The separation of states into regular and irregular ones becomes fully explicit in our second catalogue of states. The catalogue consists of 100 consecutive states starting at the consecutive index of about $2.5 \cdot 10^{6}$. These states were obtained by the scaling method first introduced by Vergini and Saraceno (1995), that enables us to find a few states in the neighbourhood of a chosen wavenumber $k$. As this is a diagonalizational method no levels were missed. Almost each level in our small catalogue can be clearly identified as regular or irregular, an idea proposed already by Percival (1973). The only exception in the catalogue is a pair of states lying close together with respect to the mean level spacing, shown in figure 8, where both of the states are superpositions of a regular $\left(\left|\psi_{r}\right\rangle\right)$ and irregular $\left(\left|\psi_{i}\right\rangle\right)$ state. These two states are close to a degeneracy of two energetically equal but structurally different quantized classical objects.

This mixing is the consequence of the fact that the regular and irregular state are not exact solutions of the Hamiltonian which leads to the matrix element

$$
H_{r i}=\left\langle\psi_{r}|\hat{H}| \psi_{i}\right\rangle
$$

not being equal to 0 . When two such states, or, more precisely, their adiabatically corresponding states, are brought close together on the energy scale by varying a parameter of the system (e.g. $\lambda$ in our billiard), their eigenenergies do not cross but show a phenomenon of level repulsion (avoided crossing). 
In the cases when the effect of other levels can be neglected, the smallest energy spacing between the two levels reached is twice the value of the matrix element (6). At this point the eigenstates are exactly the symmetric and antisymmetric superpositions of the well-separated states. If we vary the parameter of the system further, the adiabatic equivalents of the original states will exchange identities.

While the avoided level crossings are typical for irregular states, the regular states do not exhibit the phenomenon of level repulsion amongst themselves (Berry 1983), except possibly on an exponentially small scale (due to the tunneling effects). Furthermore, according to the PUSC, there should be no level repulsion between the regular and irregular states as $\hbar \rightarrow 0$. As this repulsion is directly connected to the mixing of states, the relative number of mixed states, such as the pair shown in figure 8, is expected to tend to 0 when effective Planck's constant of the system tends to 0 .

More states of both the regular and irregular type from this catalogue with their appropriate analysis will be shown in the next section.

\section{Analysis of states}

The main purpose of our work was to understand to what extent it is possible to describe the structure of individual eigenstates by semiclassical methods. For completely integrable systems there exists the Einstein-Brillouin-Keller method of torus quantization (see for example Berry 1983, Robnik 1998), where each semiclassical eigenstate is spanned by an invariant torus in classical phase space, for which the classical actions are integer multiples of $\hbar$ with corrections due to the singularities of projection of the torus onto the configuration space. For fully chaotic systems, and general mixed systems (generic systems), the Gutzwiller periodic orbit theory (Gutzwiller 1990) can in principle be employed to obtain the density of states (the spectrum) and the wavefunctions, to the leading semiclassical approximation.

The semiclassical methods cannot, however, predict individual energy levels within a vanishing fraction of the mean level spacing even in the limit $\hbar \rightarrow 0$ (Prosen and Robnik 1993a, Robnik and Salasnich 1997). This limits their use in the analysis of statistical properties of spectra. Furthermore, in the chaotic case the levels are also very sensitive to perturbations of the system (Percival 1973). The Gutzwiller approach and method is very useful in 
the qualitative analysis, and in certain context also quantitative (in describing the collective and statistical properties), but it is still just the leading term in a certain semiclassical expansion, not good enough to resolve the fine structures with sufficient accuracy to make the analysis of individual states and energy levels reliable. Therefore, because of the sensitivity of chaotic eigenstates and the approximating nature of the theory, to some extent the questions about the fine structure of individual chaotic states are irrelevant. It is therefore more appropriate to discuss the statistical properties of chaotic states, which are, however, less sensitive to perturbations.

In a mixed system the phase space is divided into chaotic and regular components. Our work was guided by the principle of uniform semiclassical condensation (PUSC, see Robnik 1998), stating that when $\hbar$ tends to 0 the Wigner function of any eigenstate uniformly condenses on an invariant object in phase space. This can be either a torus in the regular region or a whole chaotic component. Each state could thus be labeled as either regular or irregular (chaotic) in the semiclassical limit. By looking at the catalogue of states at high (and to some extent even at low) energies, one can see that this can indeed be done, though there is still the localization phenomenon present due to the still insufficiently low value of the effective Planck's constant.

\subsection{Regular states}

We start the analysis by considering the regular states. These are the states that can be attributed to quantized tori within the regular regions. For these states we tried to employ the EBK torus quantization. We construct a wavefunction on the torus as a sum of contributions

$$
\psi_{j}(\mathbf{q})=A_{j}(\mathbf{q}) \exp \left(\frac{i}{\hbar} S_{j}^{c l}(\mathbf{q})+\phi_{j}\right)
$$

of different projections $j$ of the torus onto configuration space. $S_{j}$ is the classical action with respect to some point on the torus and $A_{j}^{2}$ the classical density of trajectories on this projection. The phase of the wavefunction must change by an integer multiple of $2 \pi$ when going around any closed contour of the torus. This gives us the quantization conditions

$$
I_{i}=\frac{1}{2 \pi} \oint_{\gamma_{i}} \mathbf{p} \cdot d \mathbf{q}=\hbar\left(n_{i}+\beta_{i} / 4\right)
$$


where $\gamma_{i}$ are the irreducible closed contours on the torus and $n_{i}$ the torus quantum numbers. The integers $\beta_{i}$ are Maslov's corrections and arise due to the changes of phase $\phi_{j}$ at the singularities of projection of the torus onto configuration space. At each caustic encountered along the contour $\gamma_{i}$ the wavefunction acquires a negative phase shift of $\pi / 2$, and shifts by $\pi$ when reflected from a hard wall'? From this consideration it follows that $\beta_{i}$ counts the number of caustics plus twice the number of hard walls encountered along the contour.

The main problem arises since, unlike in many completely integrable cases, the transformation to action-angle variables for regular components of mixed type systems is usually not known. The object we are dealing with is only the numerically calculated trajectory, so we must make the best of it.

The task of finding the semiclassical EBK wavefunctions can be divided into two parts. The first one is finding the torus with the desired quantum numbers $n_{i}$, the second one being the construction of its appropriate wavefunction in configuration space.

In our billiard system all the regular tori except the whispering gallery ones wind around a stable periodic orbit with a finite number of bounces $l_{b}$. We choose $\theta_{1}$ to represent the movement of the trajectories along the corresponding periodic orbit while $\theta_{2}$ represents the winding of the trajectories around it. The Maslov index for the contour along $\theta_{2}$ is $\beta_{2}=2$ since there are two singularities of projection (caustics) encountered, while for the contour along $\theta_{1}$ this index is equal to $\beta_{1}=2 l_{b}$. In the case of whispering gallery modes one similarly obtains $\beta_{1}=0$ and $\beta_{2}=3$, if $\theta_{1}$ is taken along the boundary of the billiard and $\theta_{2}$ 'perpendicular' to it.

We may find the appropriate torus by iteration. We start with a trajectory $\eta$ in a regular island on the SOS and follow it sufficiently long until it returns to the desired neighbourhood of the initial point, thus approximately completing $N_{1}$ integer number of cycles in $\theta_{1}$ and $N_{2}$ in $\theta_{2}$ in the time $T$. One can obtain the numbers $N_{1}$ and $N_{2}$ by knowing the total number of bounces $N_{b}$ and $N_{c}$ of the caustics encountered during the process through the topological properties of the torus. For a torus winding around a periodic orbit, $N_{1}=N_{b} / l_{b}$ and $N_{2}=N_{c} / 2$. The winding frequencies on the torus are

\footnotetext{
${ }^{2}$ If the contour passes the singularity in the contrary direction to that of the Hamiltonian flow on the torus, the phase shifts are of the opposite sign
} 
then given by

$$
\omega_{i}=2 \pi N_{i} / T \text {. }
$$

Finding the number of bounces $N_{b}$ along the trajectory is straightforward. The number of caustics $N_{c}$ may be obtained by starting a trajectory $\eta^{\prime}$ near the original trajectory $\eta$ on the same torus (more are needed in case of more than two dimensions). Whenever the two trajectories cross there is a singularity of the density of trajectories and hence a caustic.

One could of course use the monodromy matrix to find the caustics. There are two reasons for not doing so. The first one is the numerical simplicity of our approach. The more important reason is, however, that if the trajectories $\eta$ and $\eta^{\prime}$ are taken too close together (infinitesimally close together in the monodromy matrix approach) we may observe caustics due to the possible graining of the desired torus into smaller islands of stability, which quantum mechanics is at the given value of effective Planck's constant still unable to resolve. A good criterion is that the trajectories should be separated by $\approx 1 / n_{i}$ for each $\theta_{i}$

We still have to calculate the action integrals on the torus. The integral along $\theta_{2}$ can be calculated by the integral

$$
I_{2}=\frac{1}{2 \pi} \oint_{\gamma_{2}} \mathbf{p} \cdot d \mathbf{q}
$$

along the curve $\gamma_{2}$ formed by the crossing points of the trajectory with the SOS. This integral is just $1 / 2 \pi$ of the area of the intersection of the torus with the SOS. Another action integral we can calculate is the action along the chosen orbit $\eta$,

$$
I=\frac{1}{2 \pi} \int_{\eta} \mathbf{p} \cdot d \mathbf{q}
$$

This integral is the sum of $I=N_{1} I_{1}+N_{2} I_{2}$, so the integral along $\theta_{2}$ is equal to

$$
I_{1}=\left(I-N_{2} I_{2}\right) / N_{1} .
$$

We must iterate this procedure by choosing different starting points until the proper torus fulfilling the conditions (8) has been found. In general there are as many conditions as there are degrees of freedom. For two dimensional billiard systems whose dynamics is independent of energy, all action integrals can be written in the form

$$
I_{m}=G_{m} \sqrt{E}
$$


where $G_{m}$ is an integral dependent purely upon the geometry of the classical object in question. In order to obtain the correct quantized torus, instead of solving two separate equations, due to this scaling property we need only to find the appropriate ratio of the (geometric) actions

$$
\frac{I_{1}}{I_{2}}=\frac{n_{1}+\beta_{1} / 4}{n_{2}+\beta_{2} / 4}
$$

We used the robust bisection method to fulfill this condition since the dependence of actions upon initial conditions is not smooth due to the previously mentioned graining of the tori. Note that this procedure yields only the correct geometry of the quantized torus, the energy of which must still be determined by the quantization conditions 8 .

The classical action as a function of time can then be written as

$$
\int_{\mathbf{q}_{0}}^{\mathbf{q}(t)} L(\mathbf{q}, \dot{\mathbf{q}}) d t=S(t)=\left(I_{1} \omega_{1}+I_{2} \omega_{2}\right) t
$$

so one immediately obtains the semiclassical energy as

$$
E=\frac{\partial S}{\partial t}=I_{1} \omega_{1}+I_{2} \omega_{2}
$$

In our case we took the quantized values for $I_{1}$ and $I_{2}$ and taken into account that for billiard systems the angular frequencies are of the form

$$
\omega_{m}=\lambda_{m} \sqrt{E}
$$

where $\lambda_{m}$ are frequencies dependent again only upon the geometry of the chosen torus.

In principle one could obtain the semiclassical energy without considering the angular frequencies $\omega_{i}$ by simply finding the appropriate torus through the quantization conditions 8 and reading its energy. In a KAM system such as ours, however, these quantization conditions may be only approximately fullfiled due to the fine structure of the phase space. In contrast to the actions $I_{i}$ the frequencies $\omega_{i}$ do not depend as strongly on the initial conditions (close to the main periodic orbit of an island of stability the transversal frequency typically does not vanish but is characteristic of the periodic orbit). So by taking the quantized values for actions $I_{i}$ and the numerical values for $\omega_{i}$ in equation (16) the best estimate of the semiclassical energy is obtained. 
Once the proper torus has been found, we proceed to the second step. We need to span the semiclassical wavefunction (7) on this torus. Again we deal only with a trajectory, so we must find means of representing the wavefunction with it. We may write the wavefunction in the form

$$
\psi_{j}(\mathbf{q})=\lim _{T \rightarrow \infty} 1 / T \int_{0}^{T} d t \delta\left(\mathbf{q}_{c l}(t)-\mathbf{q}\right) D_{j}\left(\mathbf{q}_{c l}(t)\right) \exp \left(\frac{i}{\hbar} S_{j}\left(\mathbf{q}_{c l}(t)+\phi_{j}\right)\right.
$$

where $\mathbf{q}_{c l}(t)$ is the classical trajectory in configuration space. This definition is appropriate only in the sense of integrals of the wavefunction over configuration space. We can hence determine the function $D_{j}$ by integrating the wavefunction over a small volume $V^{\prime}$,

$$
\int_{V^{\prime}} d V \psi_{j}=\lim _{T \rightarrow \infty} D_{j}(\mathbf{q}) \exp \left(\frac{i}{\hbar} S_{j}(\mathbf{q})+\phi_{j}\right) \frac{T^{\prime}}{T}
$$

where $T^{\prime}$ is the time the trajectory spends in the volume $V^{\prime}$. This time is proportional to the density of trajectories $A_{j}^{2}$ in the volume $V^{\prime}$,

$$
T^{\prime}=T A_{j}^{2}(\mathbf{q}) V^{\prime} .
$$

If we want the expression (19) to be consistent with (7),

$$
D_{j}=\frac{1}{A_{j}}
$$

must hold.

We still have to account for the phase shifts when the trajectory traverses from one projection of the torus onto another. This can be done hand in hand with the estimation of the density of trajectories $A_{j}^{2}$. Again we start a trajectory $\mathbf{q}_{c l}^{\prime}$ close to the original one (more trajectories are needed in more than two degrees of freedom). We could again use the monodromy matrix approach, but the same criticism applies here as in the case of finding the appropriate tori. Let us imagine a bundle of trajectories inside a small parallelogram spanned by the three points $\mathbf{q}_{c l}, \mathbf{q}_{c l}^{\prime}$ and $\mathbf{q}_{c l}+\dot{\mathbf{q}}_{c l} \delta t$, where all three points lie on the given torus. The area of the parallelogram is given by the absolute value of

$$
\mathbf{P}=\dot{\mathbf{q}}_{c l} \times\left(\mathbf{q}_{c l}^{\prime}-\mathbf{q}_{c l}\right) \delta t
$$


As we are interested only in the relative sizes of this parallelogram, we may set $\delta t=1$. The reciprocal value of this area is proportional to the density of trajectories,

$$
A_{j}^{2}=\alpha /|P| .
$$

The value of $\alpha$ does not change along the trajectory and can be subsequently determined by the normalization of the semiclassical wavefunction. Whenever the value of $P$ changes its sign, the trajectory has encountered a caustic and has passed from one projection $j$ of the torus to another one $k$. At this point the wavefunction acquires a phase shift

$$
\phi_{k}=\phi_{j}-\pi / 2 \text {. }
$$

The phase shift is equal to $\pi$ if a hard wall is encountered.

The construction (18) is, in its original form, of course unsuitable for numerical computation. We must substitute the $\delta$ function in the integral by a function of finite width. The other important reason for doing so is to again smooth the classical behaviour which can have a far more detailed structure than the quantum mechanics can yet resolve. This width may not necessarily need be isotropic. A good estimate for it is again to be of the order of $1 / n_{i}$ in coordinates $\theta_{i}$ projected onto configuration space.

We used a Gaussian for the wide delta function. If we adjusted the width and the amplitude of the Gaussian so that it followed the classical density of trajectories, a remarkable similarity of our method with that of Heller's wavepacket approach was observed (Heller 1991). There are, however, two important differences. The wavepacket approach starts with a wavepacket with the expected value of energy equal to that of the exact wavefunction and then tries to construct its semiclassical approximation. Our approach is independent of the exact eigenstate. All the information we have to supply is the quantum numbers and the geometrical properties of the (projection of the) torus, obtaining from them both the semiclassical energy (16) and the wavefunction. The other important difference is that the wavepacket approach relies on the monodromy matrix of the trajectory, the use of which can be questionable to obtain semiclassical wavefunctions in mixed type systems due to the fine structure of the classical phase space, as was already pointed out before.

We show three examples of the regular states in the figures 9 to 11. For each of the states we present the exact numerical quantum probability density(top left), the probability density of its semiclassical approximation (top 
right), the classical density of trajectories on the appropriate torus (bottom left) and the smoothed projection of the exact Wigner function (bottom right). The semiclassical wavefunctions shown are remarkable as they possess all of the features of their exact counterparts that are larger than the appropriate wavelength. Note that for each torus there are two characteristic wavelengths since there are two quantum numbers associated with it. As it happens in our case, the two wavelengths can be of different orders of magnitude.

There were, however, some 'regular' states that we were unable to semiclassically reproduce. The first class of these states can be described as localized chaotic states since their Wigner function clearly shows that they lie in the chaotic region, yet very close to an island of stability, which gives them a regular appearance. The other class are the states whose Wigner transforms lie in the regular regions, but where the primary tori have already been destroyed by the perturbation and now form secondary tori interwoven by small regions of chaotic motion, and even the smoothing of classical dynamics, as described above, fails.

The accuracy of the semiclassical energies that we were indeed able to reproduce may seem remarkable, since the error is approximately 5 units of energy at the energies around $2 \cdot 10^{7}$. Such accuracy, however, is still insufficient to perform short range spectral statistics since the mean level spacing in our system is approximately 8 units of energy. This experience is of course in agreement with the proposition and conclusion that the semiclassical methods (to the leading order) cannot resolve the energy spectra within the vanishing fraction of the mean level spacing, and also not the structures of the wavefunctions smaller than de Broglie wavelength (Prosen and Robnik 1993a, Robnik and Salasnich 1997).

\subsection{Irregular states}

While for the regular states it was quite straightforward to find their semiclassical approximations, the nature of irregular states is very much different. The chaotic component of a system does not possess any obvious structure. While Gutzwiller's approach can yield the properties of a quantum system by a summation over all periodic orbits of its classical counterpart, the relevance of examining individual chaotic states becomes questionable. These states are very sensitive to small perturbations of the system, so in any physical sys- 
tem the individual features of the states are lost when the effective Planck's constant tends to 0 . The features that are insensitive to small perturbations are, however, the statistical properties of spectra and eigenstates.

One measure of statistical properties of the wavefunctions is the wavefunction autocorrelation function,

$$
C(\mathbf{q}, \mathbf{x})=\frac{\left\langle\psi^{\dagger}\left(\mathbf{q}^{\prime}-\mathbf{x} / 2\right) \psi\left(\mathbf{q}^{\prime}+\mathbf{x} / 2\right)\right\rangle_{\mathbf{q}^{\prime} \in \epsilon(\mathbf{q})}}{\left\langle\psi^{\dagger}\left(\mathbf{q}^{\prime}\right) \psi\left(\mathbf{q}^{\prime}\right)\right\rangle_{\mathbf{q}^{\prime} \in \epsilon(\mathbf{q})}} .
$$

The area of averaging $\epsilon(\mathbf{q})$ close to the point $\mathbf{q}$ should be taken such that its linear size is many wavelengths across, however small enough that the local properties of classical mechanics within it are largely uniform.

If one takes the Fourier transform of the Wigner function (3), it is easy to show that

$$
\int W(\mathbf{q}, \mathbf{p}) \exp (i \mathbf{p} \cdot \mathbf{x} / \hbar) d^{N} \mathbf{p}=\psi^{\dagger}(\mathbf{q}-\mathbf{x} / 2) \psi(\mathbf{q}+\mathbf{x} / 2) .
$$

By knowing the Wigner function of an eigenstate, it is then possible to use this result to calculate its autocorrelation function.

According to the principle of uniform semiclassical condensation, the Wigner function of any chaotic state should uniformly condense on the whole chaotic component when the effective $\hbar$ tends to 0 . Let us limit ourselves only to the cases of the Hamiltonians with an isotropic dependence upon $\mathbf{p}$. We can write the semiclassical Wigner function in the form of a conditional delta function

$$
W_{\mathcal{D}_{i}}(\mathbf{q}, \mathbf{p})=\alpha \delta\left(\{\mathbf{q}, \mathbf{p}\} \in \mathcal{D}_{i} ; E-H(\mathbf{q}, p)\right)
$$

where $\mathcal{D}_{i}$ denotes a chaotic component and $\alpha$ is the normalization constant. The Fourier transform (26) of this Wigner function is

$$
\int W_{\mathcal{D}_{i}}(\mathbf{q}, \mathbf{p}) p^{N-1} \exp (i \mathbf{p} \cdot \mathbf{x} / \hbar) d p d \Omega_{p}=\alpha \frac{p(\mathbf{q})^{N-1}}{\frac{\partial H}{\partial p}(\mathbf{q}, p(\mathbf{q}))} \int_{\Omega_{p} \in \mathcal{D}_{i}(\mathbf{q})} d \Omega_{p} \exp (i \mathbf{p} \cdot \mathbf{x} / \hbar),
$$

where $p(\mathbf{q})$ denotes the absolute value of momentum at the point $\mathbf{q}$. The integration over the spatial angle $\Omega_{p}$ is performed along all the directions of momentum that constitute the chaotic component $\mathcal{D}_{i}$ at the point $\mathbf{q}$. The autocorrelation function is then equal to

$$
C_{\mathcal{D}_{i}}(\mathbf{q}, \mathbf{x})=\frac{\left\langle\int_{\Omega_{p} \in \mathcal{D}_{i}\left(\mathbf{q}^{\prime}\right)} d \Omega_{p} \exp (i \mathbf{p} \cdot \mathbf{x} / \hbar)\right\rangle_{\mathbf{q}^{\prime} \in \epsilon(\mathbf{q})}}{\left\langle\int_{\Omega_{p} \in \mathcal{D}_{i}\left(\mathbf{q}^{\prime}\right)} d \Omega_{p}\right\rangle_{\mathbf{q}^{\prime} \in \epsilon(\mathbf{q})}}
$$


The averaging area should again stretch across many wavelengths.

If the chaotic component is equal to the whole energy surface, as is the case in completely ergodic systems, in the case of two degrees of freedom one obtains the well known Berry's result (Berry 1977)

$$
C_{\text {ergodic }}(\mathbf{x})=J_{0}(p(\mathbf{q}) r / \hbar), r=|\mathbf{x}| \text {. }
$$

However, when the system is of the mixed type the autocorrelation function ceases to be isotropic and acquires contributions of higher order Bessel functions. We can obtain these contributions by rewriting integrals $\int_{\phi_{p} \in \mathcal{D}_{i}} f\left(\phi_{p}\right) d \phi_{p}$ by integrals of the characteristic function $\int \chi_{\mathcal{D}_{i}}\left(\phi_{p}\right) f\left(\phi_{p}\right) d \phi_{p}$, where in two degrees of freedom the spatial angle is replaced by a simple angle $\phi_{p}$ and $f\left(\phi_{p}\right)$ is an arbitrary function of $\phi_{p}$. If we write the characteristic function as a Fourier series,

$$
\chi_{\mathcal{D}_{i}}\left(\mathbf{q} ; \phi_{p}\right)=\sum_{m=-\infty}^{\infty} \kappa_{m}^{\mathcal{D}_{i}}(\mathbf{q}) \exp \left(i m \phi_{p}\right)
$$

it is quite straightforward to show by using the integral representations of the Bessel functions that

$$
C_{\mathcal{D}_{i}}(\mathbf{q}, \mathbf{x})=\frac{\left\langle\sum_{m=-\infty}^{\infty} \kappa_{m}^{\mathcal{D}_{i}}\left(\mathbf{q}^{\prime}\right) i^{m} J_{m}\left(p\left(\mathbf{q}^{\prime}\right) r / \hbar\right) \exp \left(i m \phi_{x}\right)\right\rangle_{\mathbf{q}^{\prime} \in \epsilon(\mathbf{q})}}{\left\langle\kappa_{0}^{\mathcal{D}_{i}}\left(\mathbf{q}^{\prime}\right)\right\rangle_{\mathbf{q}^{\prime} \in \epsilon(\mathbf{q})}}
$$

where $\phi_{x}$ is the polar angle of the vector $\mathbf{x}$.

As in the case of the regular states, numerically we cannot deal with components of phase space but with trajectories. So we start a trajectory within the chaotic component $\mathcal{D}_{i}$ and not the direction of its momentum $\phi_{p}^{j}(\mathbf{q})$ at each passage $j$ through the neighbourhood of the point $\mathbf{q}$. The averaged characteristic function for this neighbourhood can then be represented as

$$
\chi_{\mathcal{D}_{i}}\left(\mathbf{q} ; \phi_{p}\right) / \kappa_{0}^{\mathcal{D}_{i}}(\mathbf{q})=\frac{\sum_{j=1}^{n} d_{j} \delta\left(\phi_{p}-\phi_{p}^{j}\right)}{\sum_{j=1}^{n} d_{j}},
$$

where $d_{j}$ are the lengths covered by the particle in the averaging neighbourhood at the passage $j$, and $n$ is the number of passages. One can check that this method tends to the proper angular distribution $\chi_{\mathcal{D}_{i}}$ as $n \rightarrow \infty$ for any shape of the averaging neighbourhood, if one assumes the homogeneity of trajectories at a given angle (which is true for a small enough neighbourhood, 
over which the classical phase space picture does not vary). For any incident angle $\phi$ the conditional expected value of a contribution to the equation (33) is proportional to $\int d_{j} d x$, where $x$ is the homogeneously distributed impact parameter (the direction perpendicular to the incident angle). This integral gives just the area of the averaging neighbourhood and is clearly the same for all incident angles.

If the area of averaging is large enough so that the variations in the classical phase space picture become important, the above procedure is still valid as long as the value of the momentum does not change appreciably (as is the case in our billiard system, where between the bounces the momentum remains constant). One can then imagine the large averaging area as being cut into smaller ones within which the above assumptions still hold true.

We compared the autocorrelation functions for a few chaotic states with the semiclassical prediction in figures 12 to 14 . The averaging area $\epsilon(\mathbf{q})$ was taken as a circle of radius 0.2 around the point $(x, y)=(0.65,0)$ (the coordinates are as defined in equation (1)). It was taken the same for both the semiclassical prediction and for the numerical results. The averaging radius was taken quite large in order to reduce the localization properties of the wavefunctions, which are still apparent at the values of effective $\hbar$ that we were able to obtain. But this radius still has to be taken small enough in order not to completely smooth out the classical dynamics. The agreement with the semiclassical prediction is quite good particularly in figure 14. In all cases it clearly deviates from the Berry's prediction for fully ergodic systems (30), as it must for mixed systems, and tends towards our semiclassical result. Although in some cases there are amplitude deviations from our prediction, in all of the plots the phase of the numerical correlation function matches the phase of its semiclassical prediction and is significantly different from the phase predicted for the fully ergodic case.

\section{Discussion and conclusion}

As already presented by Prosen and Robnik (1993c), the classification of states into regular and irregular ones is well founded when the effective Planck's constant tends to 0 . Its theoretical foundation is the principle of uniform semiclassical condensation of Wigner functions of eigenstates (Robnik 1988, 1998). This separation is not strictly a semiclassical phenomenon 
since even some of the lowest levels in our catalogue of low lying states can be classified as either regular or irregular. In the high energy catalogue of states each state can easily be classified as either chaotic or regular, with only one notable exception, where two close lying states are a superposition of a regular and an irregular state. These exceptions are expected to disappear with higher energies. While the states can be separated with respect to classical dynamics, the chaotic states in our high energy catalogue still exhibit the phenomenon of dynamical quantal localization. Their Wigner functions are not uniformly extended over the whole chaotic component, but are significant only on a part of it. This localization is expected to disappear at sufficiently small effective $\hbar$, when the quantum mechanical break time $t_{\text {break }}=\hbar / \Delta E$, where $\Delta E$ is the mean level spacing, becomes longer than the time for a typical trajectory to explore the whole chaotic component (diffusion time).

The most important part of this work is of course the semiclassical analysis of states. We were able to reconstruct both the semiclassical wavefunction and the semiclassical energy of the regular states by using the EBK quantization. Since our system is of a KAM type, most of the resonant tori are destroyed, forming smaller islands of stability interwoven with chaotic components. The classical mechanics thus shows a rich structure that quantum mechanics at a fixed value of $\hbar$ is still unable to resolve. In order to obtain the regular semiclassical wavefunctions, we had to appropriately smooth out this fine classical behaviour as explained in the section 3.1.

We were unable to predict the individual properties of chaotic states. We made a step forward, however, in describing their semiclassical statistical properties. We obtained a semiclassical prediction for the autocorrelation function of their wavefunction, which differs from the one for fully ergodic systems as it is not isotropic. The numerical results confirm this prediction, although there are still localization phenomena at the currently attainable effective $\hbar$ that cause deviations from it.

One aspect that needs to be investigated further is the localization properties of the chaotic states. As Casati and Prosen (1998) show in the example of a fully chaotic stadium billiard, in the diffusive regime $(\epsilon$-stadium, having very large ergodic time), the quantum diffusion is stopped by the cantori in phase space leading to localization. How these and similar ideas translate to the case of a mixed type system remains so far an open question. 


\section{Acknowledgments}

We thank Dr. Tomaž Prosen for assistance and advise with some computer programs. This work was supported by the Ministry of Science and Technology of the Republic of Slovenia and by the Rector's Fund of the University of Maribor. 


\section{References}

Berry M V 1977, J. Phys. A: Math. Gen. 102083

Berry M V 1983 Chaotic Behaviour of Deterministic Systems (Proc. NATO ASI Les Houches Summer School ed G Iooss, R H G Helleman, R Stora (Amsterdam: Elsevier) p 171

Berry M V and Robnik M 1984 J. Phys. A: Math. Gen. 172413

Berry M V and Wilkinson M 1984 Proc. Roy. Soc. London A 39215

Bogomolny E 1988 Physica 31D 169

Casati G and Prosen T 1999 Phys. Rev. E 592516

Gutzwiller M 1990, Chaos in Classical and Quantum Mechanics, Springer New York

Heller E J 1984 Phys. Rev. Lett. 531515

Heller E J 1986 Lecture Notes in Physics 263162

Heller E J 1991 Chaos and Quantum Physics (Proc. NATO ASI Les Houches Summer School ed M-J Giannoni, A Voros and J Zinn-Justin (Amsterdam: Elsevier) p 547

Lazutkin V F 1981 The Convex Billiard and the Eigenfunctions of the Laplace Operator (Leningrad: University Press) (in Russian)

Lazutkin V F 1991 KAM Theory and Semiclassical Approximations to Eigenfunctions (Heidelberg: Springer)

Li Baowen and Robnik M 1994 J. Phys. A: Math. Gen. 275509

Li Baowen and Robnik M 1995a J. Phys. A: Math. Gen. 282799 
Li Baowen and Robnik M 1995b J. Phys. A: Math. Gen. 284483

Percival I C 1973 J. Phys. B: At. Mol. Phys. 6 L229

Prosen T 1995 J. Phys. A: Math. Gen. 28 L349

Prosen T 1996 Physica D 91244

Prosen T 1998 J. Phys. A: Math. Gen. 347023

Prosen T and Robnik M 1993a J. Phys. A: Math. Gen. 26 L37

Prosen T and Robnik M 1993b J. Phys. A: Math. Gen. 262371

Prosen T and Robnik M 1993c J. Phys. A: Math. Gen. 265365

Prosen T and Robnik M 1994 J. Phys. A: Math. Gen. 278059

Prosen T and Robnik M 1999 J. Phys. A: Math. Gen. 321863

Robnik M 1983 J. Phys. A: Math. Gen. 163971

Robnik M 1984 J. Phys. A: Math. Gen. 171049

Robnik M 1988 in Atomic Spectra and Collisions in External Fields Eds. K.T. Taylor, M.H. Nayfeh and C.W. Clark (New York: Plenum Press) pp251274

Robnik M 1989 Bound-State Eigenfunctions of Classically Ergodic Hamilton Systems: A Theory of Scars Preprint Institute for Theoretical Physics, University of California Santa Barbara, unpublished

Robnik M 1998 Nonlinear Phenomena in Complex Systems 11

Robnik M and Salasnich L 1997 J. Phys. A: Math. Gen. 301711

Robnik M and Veble G 1998 J. Phys. A: Math. Gen. 314669 
Vergini E and Saraceno M 1995 Phys. Rev. E 522204

Voros A 1979 Lecture Notes in Physics 93326 
Figure 1: The SOS section of the main chaotic component of the $\lambda=0.15$ billiard. The coordinate $x$ here is shifted by $\lambda$ to the right so that $x \in$ $[-1,+1]$, and $x=0$ corresponds to $x=\lambda$ of equation (1).

Figure 2: The shortest periodic orbits of the $\lambda=0.15$ billiard (explained in text). The stable orbits are shown with full and the unstable ones with dashed lines.

Figure 3: A selection of eigenstates corresponding to the stable periodic orbits 1, 2, 3, 4 in the figure 2 from top to bottom, respectively. In the top half of each plot we show the eight equally spaced contours of the probability density from 0 to its maximum value. In the bottom half we show the nodal lines of each wavefunction.

Figure 4: A selection of eigenstates corresponding to the stable periodic orbits 5 and 6 in the figure 2 in the top and bottom row, respectively. In the top half of each plot we show the eight equally spaced contours of the probability density from 0 to its maximum value. In the bottom half we show the nodal lines of each wavefunction.

Figure 5: The corresponding smoothed projections of the Wigner functions for the wavefunctions in the figure 3. The contours are spaced in ten intervals from 0 to the maximal value. The negative value contours are plotted at the same spacing but with thinner lines. We do not plot the zero level contour due to many oscillations of the Wigner functions when it is close to zero. As in all subsequent Wigner plots, the momentum $p_{x}$ is measured in units of $p^{\prime}$, which is related to the energy of the eigenstate by $E=p^{\prime 2}$. The locations of the relevant classical periodic orbits are marked by the "bullets". The coordinate $x$ here is shifted by $\lambda$ as in figure 1 .

Figure 6: The corresponding smoothed projections of the Wigner functions for the wavefunctions in the figure 1 . The plotting method is the same as in figure 5 . 
Figure 7: An example of the probability density for a regular (top left) and an irregular (top right) state with the corresponding smoothed projections of their Wigner functions shown below, with the same plotting methods as used in corresponding previous plots. In the phase space plots (bottom row) the coordinate $x$ is shifted by $\lambda$ as in figure 1 . The "bullets" mark the location of the stable period 3 periodic orbit, which is the skeleton of the quantized invariant torus.

Figure 8: The probability density (16 equally spaced contours from 0 to the maximum value) for a pair of close lying states $\left(k^{2}=20421106.7347\right.$ left and $k^{2}=20421107.0691$ right) that are superpositions of a regular and an irregular state.

Figure 9: The probability density for a regular state with $k^{2}=20420831.0603$ (top left), its semiclassical approximation with $k_{s c}^{2}=20420828.18$ (top right), with the quantum numbers on the torus being $n_{1}=6752$ and $n_{2}=37$ (16 equally spaced contours from 0 to the maximum value in both cases). In the bottom row we show the classical density with 20 contours from 0 to the maximum of the appropriate torus (left) and the smoothed projection of the exact Wigner function (right), with ten contours from 0 to the maximum value. In the phase space plot (bottom right) the coordinate $x$ is shifted by $\lambda$ as in figure 1.

Figure 10: The same as in figure 9 but for the state with $k^{2}=20421002.7443$ and its semiclassical approximation with $k_{s c}^{2}=20420999.46, n_{1}=2861$ and $n_{2}=20$.

Figure 11: The same as in figure 9 but for the state with $k^{2}=20421387.1741$ and its semiclassical approximation with $k_{s c}^{2}=20421385.96, n_{1}=8648$ and $n_{2}=1$. 
Figure 12: In the top row we show the probability density for the chaotic state with $k^{2}=20420756.1273$ (left, 8 contours) with the smoothed projection of its Wigner function (right, 10 contours). The circle of radius 0.2 centered at $x=0.5$ is the region of averaging. In the phase space plot (top right) the coordinate $x$ is shifted by $\lambda$ as in figure 1 . In the bottom row we plot the wavefunction autocorrelation function (averaged over the small circle as explained in text) in the $x$ (left) and $y$ (right) directions.

Figure 13: The same as in figure 12 but for the state with $k^{2}=$ 20421005.3834 .

Figure 14: The same as in figure 12 but for the state with $k^{2}=$ 20421262.6667 . 
This figure "fig01.gif" is available in "gif" format from: http://arxiv.org/ps/nlin/0003061v2 
This figure "fig02.gif" is available in "gif" format from: http://arxiv.org/ps/nlin/0003061v2 
This figure "fig03.gif" is available in "gif" format from: http://arxiv.org/ps/nlin/0003061v2 
This figure "fig04.gif" is available in "gif" format from: http://arxiv.org/ps/nlin/0003061v2 
This figure "fig05.gif" is available in "gif" format from: http://arxiv.org/ps/nlin/0003061v2 
This figure "fig06.gif" is available in "gif" format from: http://arxiv.org/ps/nlin/0003061v2 
This figure "fig07.gif" is available in "gif" format from: http://arxiv.org/ps/nlin/0003061v2 
This figure "fig08.gif" is available in "gif" format from: http://arxiv.org/ps/nlin/0003061v2 
This figure "fig09.gif" is available in "gif" format from: http://arxiv.org/ps/nlin/0003061v2 
This figure "fig10.gif" is available in "gif" format from: http://arxiv.org/ps/nlin/0003061v2 
This figure "fig11.gif" is available in "gif" format from: http://arxiv.org/ps/nlin/0003061v2 
This figure "fig12.gif" is available in "gif" format from: http://arxiv.org/ps/nlin/0003061v2 
This figure "fig13.gif" is available in "gif" format from: http://arxiv.org/ps/nlin/0003061v2 
This figure "fig14.gif" is available in "gif" format from: http://arxiv.org/ps/nlin/0003061v2 